\title{
Mechanisms for optimizing procurement activities in construction based on cost management of contract works
}

\author{
Ilnur Kharisov ${ }^{1}$, Iuliia Artamonova ${ }^{2, *}$, Pavel Bilenko $^{3}$ and Sergey Sborshikov ${ }^{1}$ \\ ${ }^{1}$ Moscow State University of Civil Engineering (MGSU), 129337, 26, Yaroslavskoye Shosse, \\ Moscow, Russia \\ ${ }^{2}$ Penza State University of Architecture and Construction, 440028 Titova Street, 28, Penza, Russia \\ ${ }^{3}$ Moscow School of Management SKOLKOVO, Novaya Str., 100, Skolkovo, Moscow Region, \\ 143025, Russia
}

\begin{abstract}
The problem of optimizing procurement activities is one of the most important at the enterprises of the construction complex, since it allows saving financial resources and reducing the time of work with constant quality parameters. As a rule, based on the results of the procurement, an agreement is concluded with the supplier that offered the lowest price. This methodology does not allow evaluating the proposed price options in terms of finding reserves to reduce construction costs, since This technique does not reveal all the hidden "pitfalls" that "unscrupulous contractors" lay when conducting tenders. Often, the customer of a construction project becomes hostage to the "low price", at which it is necessary either to increase the price, or to increase the terms, or to change the contractor organization. The solution to this problem can be a new methodology for preparing tender documents, which assumes the classification of types of work in the form of a hierarchical structure and the determination of cost by type and code of work. This makes it possible to make a comparative assessment of contractors' proposals in the context of individual cost items and to determine the average price based on the results of the tender. At the second stage of the competitive procedure, contractors are asked to optimize their bids to the level of the average or the lowest price, if they are higher, and to keep the offered price, if it was the lowest. Often contractors work with "usual" suppliers and stop monitoring the market for the best price. The use of this technique leads to minimization of the costs of contracting work both for the customer at the expense of the minimum price and for the contractor by identifying the potential for price reductions, and also allows the customer to obtain the required volume of construction work of the required quality within the planned time frame without reducing the internal rate of return of the contracting company organizations.
\end{abstract}

\footnotetext{
* Corresponding author: clusterwings@mail.ru
} 


\section{Introduction}

The modern development of the economy is due to an increase in the volume of reproduction of fixed assets of the national economy, which leads to an increase in the volume of contract trading, which does not always correspond to the principles of efficiency and rationality. Nevertheless, such a system, involving the creation of competitive conditions, is one of the most effective, because it allows achieving the most favorable price-quality ratio for state and municipal authorities, balancing the positions of many suppliers on the market.

Currently, the market for construction services carried out using tendering mechanisms is not transparent. Optimization of the methodology for pricing construction work is particularly acute. Based on the results of tenders, customers choose the contractor who offered the best conditions in terms of time and price. As a result, the winners are the companies that offered the lowest price. However, the standard pricing conditions based on the base-index method differ from the real ones in the direction of increasing the cost of materials and lowering prices for construction and installation work.

This problem is caused by the fact that contractors for a long time paid wages to workers as a cash under the table understating the taxable base of the organization. Rosstat, when forming the wage index in the industry, is guided by the official data on the level of wages in the region. A paradoxical situation has developed when contractors cannot pay official salaries, since indices in the estimate for wages do not correspond to market conditions, and Rosstat cannot raise indices, since official wages are low. Also, the baseindex method is not transparent, since construction costs often depend on the qualifications of the estimator.

According to E.A. Belyaeva, conducting transparent and open public procurement is an effective way to balance the positions of many suppliers in the market [1].

The issues of pricing in the construction sector with its application in the procurement market in construction are studied in economic and economic practice. So, according to the scientific articles of V.I. Sorokina, the problem of pricing in construction should take into account market factors when determining individual elements of the value of an object, as well as when evaluating a unit of final construction products. L.V. Brezgina proposes a new mechanism for managing the cost of investment and construction projects at the stage of contract bidding. Within the framework of this mechanism, it assesses the market behavior of contract bidders, excluding unreasonable understatement of the initial contract price due to behavioral factors. This approach is justified, but the specified method, in our opinion, does not provide a sufficient level of objectivity of the assessment due to the consideration of behavioral factors.

Also is interesting the research by M.V. Demidenko, who is developing a mechanism intended for use in contract bidding, which allows to cut off applicants who have declared unreasonably low prices.

Nevertheless, all the studies cited do not take into account the objective reasons for the understatement of prices, in particular, insufficient quality assessment of the internal potential of the contractor company, leading to overestimated results of cost calculations for certain types of work. An enterprise can, by identifying reserves for reducing costs, provide itself flexibility in managing the price of the contract.

\section{Methodology}

The solution to this problem can be the use of the resource pricing technology adopted in the USA and Canada, according to the UNIFORMAT II classification, which makes it 
possible to compose and analyze the types of construction work at various stages of construction production.

The application of the methodology at the enterprise is based on the solution of the following tasks:

Conducting a comparative analysis of the structure of classification systems,

Methods for organizing and conducting competitive procedures,

Methods of scheduling and network planning,

Methods of management accounting for construction costs.

The principles of drawing up estimate documentation according to the UNIFORMAT II classification consist in the development of a complex of resource estimates based on bills of quantities and working documentation for construction. This classification is universal and can be applied to any construction site.

The classification allows you to aggregate cost items at different levels and compare them when analyzing the estimate documentation using other classification methods.

\section{Results and discussion}

There are six concepts for the placement of contracts, which can be used in the practice of enterprises in the construction industry.

Table 1. Contract placement concepts.

\begin{tabular}{|l|l|}
\hline \multicolumn{1}{|c|}{ Concept } & \multicolumn{1}{c|}{ Features: } \\
\hline $\begin{array}{l}\text { Traditional general } \\
\text { contracting }\end{array}$ & $\begin{array}{l}\text { The working draft is provided by the customer, the price is fixed. The } \\
\text { tender can be either one-stage or multi-stage. }\end{array}$ \\
\hline $\begin{array}{l}\text { Contract agreement } \\
\text { with the design } \\
\text { development of the } \\
\text { contractor }\end{array}$ & $\begin{array}{l}\text { The contractor participates in the finalization of the design } \\
\text { documentation and can either build on a turnkey basis or work on the } \\
\text { design and build principle }\end{array}$ \\
\hline Packaging works & $\begin{array}{l}\text { Works are performed in packages. Contracts are concluded either by } \\
\text { direct contracts with contractors or through a general contractor. }\end{array}$ \\
\hline $\begin{array}{l}\text { Contract with } \\
\text { payment at actual } \\
\text { costs }\end{array}$ & $\begin{array}{l}\text { The contract price is determined on the basis of prices and actual work } \\
\text { performed. }\end{array}$ \\
\hline $\begin{array}{l}\text { Contract with a } \\
\text { guarantee of the } \\
\text { maximum price of } \\
\text { work }\end{array}$ & $\begin{array}{l}\text { This type of contract is a contract with a fixed price that does not } \\
\text { change, with the exception of cases when the customer makes changes } \\
\text { to the technical documentation that entail changes in the scope of work } \\
\text { of the contractor. The guarantee of the maximum price of work is } \\
\text { provided only if the working documentation is at least 60-70\% } \\
\text { complete. } \\
\text { The advantage is confidence in the final price of the work, the } \\
\text { contractor is responsible for all unforeseen risks. }\end{array}$ \\
\hline $\begin{array}{l}\text { Contractor } \\
\text { investment contract }\end{array}$ & $\begin{array}{l}\text { Design, construction, financing and operations are usually carried out } \\
\text { in the form of partnership agreements with government agencies. }\end{array}$ \\
\hline
\end{tabular}

The search for a contractor should take into account the following factors

1) The structure and organization of companies;

2) Work experience and completed projects, as well as work experience in a country or region;

3) Availability of resources in terms of management, technical personnel, construction and production facilities, conditions for maintenance and training of personnel, as well as others related to the implementation of the project;

4) Quality assurance system; 
5) The ratio of the scope of work, transferred to the subcontract and carried out inhouse;

6) Financial stability.

If, after excluding firms that have been found to be ineligible, the list includes more than six potential bidders, and the investor is not restricted by any special rules or conditions, the selection process should continue to exclude firms that are slightly less qualified than others, and thus obtain a final list of no more than six firms.

Next, their bids are evaluated and the average price for the second stage of the tender is determined.

This methodology assumes that construction-related work must be assigned to one of three levels of the hierarchy.

Level 1 includes elements of the main group, such as underground structures, aboveground structures, internal structures, engineering networks, technological equipment, and special work.

Level 2 includes group elements, for example, foundations, basement structures, exterior decoration, roofing, load-bearing structures, stairs, plumbing, electrical, etc.,

Level 3 includes certain elements of construction and installation works, machinery, mechanisms and materials.

Determination of the cost structure for this methodology is described in Table 2.

Table 2. An example of the formation of costs by the method UNIFORMAT II.

\begin{tabular}{|l|l|l|l|l|}
\hline \multicolumn{3}{|c|}{ Cost code } & \multicolumn{1}{c|}{ Name of work elements } \\
\hline A & & & & UNDERGROUND STRUCTURES (below 0.00) \\
\hline A & 10 & & & Underground structures \\
\hline A & 10 & 00 & & Building part below 0.00 \\
\hline B & & & & SURFACE STRUCTURES (above 0.00) \\
\hline B & 10 & & & Bearing structures \\
\hline B & 10 & 00 & & Construction part above 0.00 \\
\hline D & & & & INTERNAL ENGINEERING NETWORKS \\
\hline D & 10 & & & W\&C systems, gas supply and process pipelines \\
\hline D & 10 & 20 & & Internal water supply networks (from entering the building) \\
\hline D & 10 & 30 & & Internal storm sewer networks \\
\hline D & 10 & 40 & & Internal utility sewerage networks (from the entrance to the building) \\
\hline D & 10 & 50 & & Internal gas pipeline networks \\
\hline D & 20 & & & Heating, ventilation, air conditioning systems \\
\hline D & 20 & 10 & & Heating (from entering the building) \\
\hline D & 20 & 30 & & Ventilation and air conditioning \\
\hline D & 30 & & & Fire protection systems \\
\hline D & 30 & 10 & & Fire alarm \\
\hline D & 40 & & & Power supply and lighting \\
\hline D & 40 & 00 & & Internal power supply and lighting networks \\
\hline D & 40 & 40 & & CCTV \\
\hline E & & & & EQUIPMENT \\
\hline E & 10 & & & Equipment \\
\hline E & 10 & 10 & & Industrial equipment \\
\hline E & 10 & 20 & & EPC Contractor Equipment \\
\hline E & 10 & 20 & 03 & Transport equipment \\
\hline
\end{tabular}

This classification allows you to form a hierarchical structure of work with the maximum level of detail, convenient for reading, comparison and evaluation.

Such a structure optimizes the work of managing a construction project, which makes it possible to:

1) more correct formation of value, 
2) monitoring the performance of work at various stages and adjusting the parameters of the project in the process of its implementation,

3) control over the development of financial resources in the process of project implementation and cost overruns on individual items.

The price according to the UNIFORMAT II classification must include absolutely all costs, including machines and mechanisms, procurement work, transportation costs, energy costs, cutting costs, overhead costs, estimated profit and other costs.

The basis for holding tenders must be the working documentation and the estimate drawn up in accordance with these instructions according to the UNIFORMAT II classification with the included scope of work.

The primary budgeting of the construction object is formed either at the expense of already held tender prices, or on the basis of the estimated prices of the base-index method, while one UNIFORMAT II price can be formed at the expense of several estimated prices

Table 3. Standard estimate.

\begin{tabular}{|c|c|c|c|c|c|}
\hline No & $\begin{array}{c}\text { Price code } \\
\text { and resource } \\
\text { codes }\end{array}$ & Name of work and costs & $\begin{array}{c}\text { Unit of } \\
\text { measurement }\end{array}$ & $\begin{array}{l}\text { Number } \\
\text { of units }\end{array}$ & $\begin{array}{c}\text { Total at the } \\
\text { current } \\
\text { price level, } \\
\text { rub }\end{array}$ \\
\hline 1 & 2 & 3 & 4 & 5 & 11 \\
\hline 1 & $\begin{array}{c}\text { 3.11-10-1 } \\
\text { Amendment: } \\
\text { TSN-2001.3. } \\
\text { Ex. 2. item } 3\end{array}$ & $\begin{array}{l}\text { Installation of cement } \\
\text { screeds } 20 \mathrm{~mm} \text { thick }\end{array}$ & $\begin{array}{l}100 \mathrm{~m} 2 \\
\text { screed }\end{array}$ & 54,17 & \\
\hline \multirow[t]{6}{*}{1.1} & 5745510000 & $\begin{array}{l}\text { Ready-made heavy cement } \\
\text { mortars, grade } 150\end{array}$ & м3 & 110,5068 & 0,00 \\
\hline & & Salary overhead & $\%$ & 120 & 267132,98 \\
\hline & & Estimated wage income & $\%$ & 84 & 127759,25 \\
\hline & & $\begin{array}{l}\text { Overhead and estimated } \\
\text { wage income }\end{array}$ & $\%$ & 175 & 618,94 \\
\hline & & Labor costs of key workers & man-h & 23,33 & \\
\hline & & & & & 692641,64 \\
\hline 2 & $\begin{array}{l}\text { 3.11-10-2 } \\
\text { Amendment: } \\
\text { TSN-2001.3. } \\
\text { Ex. 2. item } 3\end{array}$ & $\begin{array}{l}\text { Added or excluded for } \\
\text { every } 5 \mathrm{~mm} \text { change in the } \\
\text { screed thickness to position } \\
3.11-10-1\end{array}$ & $\begin{array}{l}100 \mathrm{~m} 2 \\
\text { screed }\end{array}$ & 54,17 & \\
\hline \multirow[t]{6}{*}{1.1} & 5745510000 & $\begin{array}{l}\text { Ready-made heavy cement } \\
\text { mortars, grade } 150\end{array}$ & $\mathrm{~m} 3$ & 27,6267 & 0,00 \\
\hline & & Salary overhead & $\%$ & 120 & 19798,22 \\
\hline & & Estimated wage income & $\%$ & 84 & 9468,71 \\
\hline & & $\begin{array}{l}\text { Overhead and estimated } \\
\text { wage income }\end{array}$ & $\%$ & 175 & 638,89 \\
\hline & & Labor costs of key workers & man-h & 0,44 & \\
\hline & & & & & 52534,52 \\
\hline
\end{tabular}

Table 4. Should look like UNIFORMAT II.

\begin{tabular}{|c|c|c|c|l|c|c|c|}
\hline A & 10 & 00 & 34 & $\begin{array}{l}\text { Installation of cement screeds, } \\
\text { 25mm thick }\end{array}$ & 5417 & $\mathrm{~m} 2$ & 745176,16 \\
\hline A & 10 & 00 & 35 & $\begin{array}{l}\text { - solution, grade according to } \\
\text { the project }\end{array}$ & 164,57 & $\mathrm{~m} 2$ & 623324,01 \\
\hline
\end{tabular}

The tender price procedure should be carried out in two stages. At the first stage of the bidding, the Bidders (subcontractors) must enter only the cost of the work (including all 
materials and temporary buildings and structures, except for the case when the Customer assumes these costs). All results are listed in Table 5.

The number of contestants must be at least three. All prices must be excluding VAT.

Table 5. Stage I tender results.

\begin{tabular}{|c|c|c|c|c|c|c|c|c|c|c|c|}
\hline 1 & 2 & 3 & 4 & $\begin{array}{l}\text { Name of } \\
\text { work } \\
\text { elements }\end{array}$ & $\begin{array}{l}\text { qt } \\
\text { y } \\
\text { pe } \\
r \\
\text { un } \\
\text { it }\end{array}$ & qty & $\begin{array}{l}\text { un } \\
\text { its } \\
\text { re } \\
\text { v }\end{array}$ & $\begin{array}{c}\text { Compet } \\
\text { itor's } \\
\text { rate No. } \\
1 \\
\text { (excludi } \\
\text { ng } \\
\text { VAT) } \\
\end{array}$ & $\begin{array}{c}\text { Compet } \\
\text { itor's } \\
\text { rate No. } \\
1 \\
\text { (excludi } \\
\text { ng } \\
\text { VAT) }\end{array}$ & $\begin{array}{c}\text { Compet } \\
\text { itor's } \\
\text { rate No. } \\
1 \\
\text { (excludi } \\
\text { ng } \\
\text { VAT) }\end{array}$ & $\begin{array}{l}\text { Avera } \\
\text { ge } \\
\text { price } \\
\text { by } \\
\text { positio } \\
\text { n }\end{array}$ \\
\hline $\begin{array}{l}\mathbf{A} \\
\mathbf{T}\end{array}$ & & & & $\begin{array}{l}\text { SURFACE } \\
\text { CONSTRUC } \\
\text { TIONS } \\
\text { (above } 0.00)\end{array}$ & & & & & & & \\
\hline $\begin{array}{l}\mathrm{A} \\
\mathrm{T}\end{array}$ & $\begin{array}{l}1 \\
0\end{array}$ & & & $\begin{array}{l}\text { Bearing } \\
\text { structures }\end{array}$ & & & & & & & \\
\hline $\begin{array}{l}\mathrm{A} \\
\mathrm{T}\end{array}$ & $\begin{array}{l}1 \\
0\end{array}$ & $\begin{array}{l}0 \\
0\end{array}$ & & $\begin{array}{l}\text { Construction } \\
\text { part above } \\
0.00\end{array}$ & & & & & & & \\
\hline $\begin{array}{l}\text { A } \\
\text { T }\end{array}$ & $\begin{array}{l}1 \\
0\end{array}$ & $\begin{array}{l}0 \\
0\end{array}$ & $\begin{array}{l}0 \\
1\end{array}$ & $\begin{array}{l}\text { Installation } \\
\text { of the metal } \\
\text { frame of the } \\
\text { building }\end{array}$ & & $\begin{array}{c}203.3 \\
3\end{array}$ & $\mathbf{t}$ & $\begin{array}{c}24,250.0 \\
0\end{array}$ & $\begin{array}{c}25,000.0 \\
0\end{array}$ & $\begin{array}{c}25,500.0 \\
0\end{array}$ & $\begin{array}{c}24,916 \\
.67\end{array}$ \\
\hline $\begin{array}{l}\mathrm{A} \\
\mathrm{T}\end{array}$ & $\begin{array}{l}1 \\
0\end{array}$ & $\begin{array}{l}0 \\
0\end{array}$ & $\begin{array}{l}0 \\
2\end{array}$ & $\begin{array}{l}\text { - metal } \\
\text { frame } \\
\text { according to } \\
\text { the project }\end{array}$ & $\begin{array}{l}1 . \\
00\end{array}$ & $\begin{array}{c}203.3 \\
3\end{array}$ & $\mathbf{t}$ & $\begin{array}{c}83,050.8 \\
5\end{array}$ & $\begin{array}{c}98,000.0 \\
0\end{array}$ & $\begin{array}{c}81,666.0 \\
0\end{array}$ & $\begin{array}{c}87,572 \\
.28\end{array}$ \\
\hline $\begin{array}{l}\mathrm{A} \\
\mathrm{T} \\
\end{array}$ & $\begin{array}{l}1 \\
0 \\
\end{array}$ & $\begin{array}{l}0 \\
0\end{array}$ & $\begin{array}{l}0 \\
3 \\
\end{array}$ & $\begin{array}{l}\text { Building } \\
\text { roofing }\end{array}$ & & $\begin{array}{c}4,535 \\
.00 \\
\end{array}$ & m2 & $3,783.00$ & $3,900.00$ & $3,822.00$ & $\begin{array}{c}3,835 \\
00\end{array}$ \\
\hline $\begin{array}{l}\mathrm{A} \\
\mathrm{T}\end{array}$ & $\begin{array}{l}1 \\
0\end{array}$ & $\begin{array}{l}0 \\
0\end{array}$ & $\begin{array}{l}0 \\
4\end{array}$ & $\begin{array}{l}\text { Monolithic } \\
\text { interfloor } \\
\text { slab device }\end{array}$ & & 38.40 & m3 & $7,566.00$ & $7,800.00$ & $7,098.00$ & $\begin{array}{c}7,488 . \\
00\end{array}$ \\
\hline $\begin{array}{l}\mathrm{A} \\
\mathrm{T}\end{array}$ & $\begin{array}{l}1 \\
0\end{array}$ & $\begin{array}{l}0 \\
0\end{array}$ & $\begin{array}{l}0 \\
5\end{array}$ & $\begin{array}{l}\text { - Concrete } \\
\text { B20, grade } \\
\text { according to } \\
\text { the project }\end{array}$ & $\begin{array}{l}1 . \\
02\end{array}$ & 39.17 & $\mathrm{~m} 3$ & $5,529.00$ & $5,700.00$ & $5,300.00$ & $\begin{array}{c}5,509 . \\
67\end{array}$ \\
\hline $\begin{array}{l}\mathrm{A} \\
\mathrm{T}\end{array}$ & $\begin{array}{l}1 \\
0\end{array}$ & $\begin{array}{l}0 \\
0\end{array}$ & $\begin{array}{l}0 \\
6\end{array}$ & $\begin{array}{l}\text { - Fittings, } \\
\text { according to } \\
\text { the project }\end{array}$ & $\begin{array}{l}0 . \\
07\end{array}$ & 2.50 & $\mathrm{t}$ & $\begin{array}{c}42,412.5 \\
0\end{array}$ & $\begin{array}{c}43,000.0 \\
0\end{array}$ & $\begin{array}{c}45,000.0 \\
0\end{array}$ & $\begin{array}{c}3,470 \\
.83\end{array}$ \\
\hline $\begin{array}{l}\mathrm{A} \\
\mathrm{T}\end{array}$ & $\begin{array}{l}1 \\
0\end{array}$ & $\begin{array}{l}0 \\
0\end{array}$ & $\begin{array}{l}0 \\
7\end{array}$ & $\begin{array}{l}\text { Installation } \\
\text { of wall } \\
\text { panels, } \\
\text { including } \\
\text { additional } \\
\text { elements }\end{array}$ & & $\begin{array}{c}4,272 \\
.40\end{array}$ & $\mathrm{~m} 2$ & $1,100.00$ & $1,000.00$ & $1,200.00$ & $\begin{array}{c}1,100 . \\
00\end{array}$ \\
\hline $\begin{array}{l}\mathrm{A} \\
\mathrm{T}\end{array}$ & $\begin{array}{l}1 \\
0\end{array}$ & $\begin{array}{l}0 \\
0\end{array}$ & $\begin{array}{l}0 \\
8\end{array}$ & $\begin{array}{l}\text { - wall panels } \\
\text { of the } \\
\text { sandwich } \\
\text { type, } \\
\text { thickness } 80 \\
\text { mm }\end{array}$ & $\begin{array}{l}1 . \\
10\end{array}$ & $\begin{array}{c}4,699 \\
.64\end{array}$ & $\mathrm{~m} 2$ & $1,697.50$ & $1,750.00$ & $1,630.00$ & $\begin{array}{c}1,692 . \\
50\end{array}$ \\
\hline $\begin{array}{l}\mathrm{A} \\
\mathrm{T} \\
\end{array}$ & $\begin{array}{l}1 \\
0\end{array}$ & $\begin{array}{l}0 \\
0 \\
\end{array}$ & $\begin{array}{l}0 \\
9 \\
\end{array}$ & $\begin{array}{l}\text { - additional } \\
\text { elements }\end{array}$ & $\begin{array}{l}0 . \\
63 \\
\end{array}$ & $\begin{array}{c}2,960 \\
.77 \\
\end{array}$ & $\mathrm{~m} 2$ & 824.50 & 850.00 & 780.00 & 818.17 \\
\hline
\end{tabular}




\begin{tabular}{|c|c|c|c|c|c|c|c|c|c|c|c|}
\hline $\begin{array}{l}\mathrm{A} \\
\mathrm{T}\end{array}$ & $\begin{array}{l}1 \\
0\end{array}$ & $\begin{array}{l}0 \\
0\end{array}$ & $\begin{array}{l}1 \\
0\end{array}$ & $\begin{array}{l}\text { Installation } \\
\text { of PVC } \\
\text { window } \\
\text { blocks } \\
\end{array}$ & & $\begin{array}{c}273.6 \\
0\end{array}$ & m2 & 508.47 & 600.00 & 460.00 & 522.82 \\
\hline $\begin{array}{l}\mathrm{A} \\
\mathrm{T}\end{array}$ & $\begin{array}{l}1 \\
0\end{array}$ & $\begin{array}{l}0 \\
0\end{array}$ & $\begin{array}{l}1 \\
1\end{array}$ & $\begin{array}{l}\text {-block } \\
\text { window }\end{array}$ & $\begin{array}{l}\text { 1. } \\
\text { 00 }\end{array}$ & $\begin{array}{c}273.6 \\
0\end{array}$ & m2 & $4,237.29$ & $5,000.00$ & $4,900.00$ & $\begin{array}{c}4,712 . \\
43\end{array}$ \\
\hline $\begin{array}{l}\mathrm{A} \\
\mathrm{T}\end{array}$ & $\begin{array}{l}1 \\
0\end{array}$ & $\begin{array}{l}0 \\
0\end{array}$ & $\begin{array}{l}1 \\
2 \\
\end{array}$ & $\begin{array}{l}\text { Can door } \\
\text { blocks }\end{array}$ & & 10.50 & m2 & 500.00 & 450.00 & 550.00 & 500.00 \\
\hline $\begin{array}{l}\mathrm{A} \\
\mathrm{T}\end{array}$ & $\begin{array}{l}1 \\
0\end{array}$ & $\begin{array}{l}0 \\
0\end{array}$ & $\begin{array}{l}1 \\
3\end{array}$ & -block door & $\begin{array}{l}1 . \\
00\end{array}$ & 10.50 & $\mathrm{~m} 2$ & $\begin{array}{c}12,000.0 \\
0\end{array}$ & $\begin{array}{c}10,500.0 \\
0\end{array}$ & $\begin{array}{c}11,000.0 \\
0\end{array}$ & $\begin{array}{c}11,166 \\
.67\end{array}$ \\
\hline $\begin{array}{l}\mathrm{A} \\
\mathrm{T}\end{array}$ & $\begin{array}{l}1 \\
0\end{array}$ & $\begin{array}{l}0 \\
0\end{array}$ & $\begin{array}{l}1 \\
4\end{array}$ & Gate can & & $\begin{array}{c}108.0 \\
0\end{array}$ & m2 & $1,000.00$ & 970.00 & $1,050.00$ & $\begin{array}{c}1,006 . \\
67\end{array}$ \\
\hline $\begin{array}{l}\mathrm{A} \\
\mathrm{T}\end{array}$ & $\begin{array}{l}1 \\
0\end{array}$ & $\begin{array}{l}0 \\
0\end{array}$ & $\begin{array}{l}1 \\
5\end{array}$ & $\begin{array}{l}\text { - gate } \\
\text { according to } \\
\text { the project }\end{array}$ & $\begin{array}{l}1 . \\
00\end{array}$ & $\begin{array}{c}108.0 \\
0\end{array}$ & $\mathrm{~m} 2$ & $\begin{array}{c}28,000.0 \\
0\end{array}$ & $\begin{array}{c}29,000.0 \\
0\end{array}$ & $2,900.00$ & $\begin{array}{c}19,966 \\
.67\end{array}$ \\
\hline $\begin{array}{l}\mathrm{A} \\
\mathrm{T}\end{array}$ & $\begin{array}{l}1 \\
0\end{array}$ & $\begin{array}{l}0 \\
0\end{array}$ & $\begin{array}{l}1 \\
6\end{array}$ & $\begin{array}{l}\text { Installation } \\
\text { of internal } \\
\text { walls and } \\
\text { partitions }\end{array}$ & & $\begin{array}{c}259.2 \\
0\end{array}$ & $\mathrm{~m} 2$ & $3,600.00$ & $3,800.00$ & $3,750.00$ & $\begin{array}{c}3,716 . \\
67\end{array}$ \\
\hline $\begin{array}{l}\mathrm{A} \\
\mathrm{T}\end{array}$ & $\begin{array}{l}1 \\
0\end{array}$ & $\begin{array}{l}0 \\
0\end{array}$ & $\begin{array}{l}1 \\
7\end{array}$ & $\begin{array}{l}\text { Finishing } \\
\text { work }\end{array}$ & & $\begin{array}{c}192.0 \\
0\end{array}$ & m2 & $4,500.00$ & $4,400.00$ & 430.00 & $\begin{array}{c}3,110 . \\
00\end{array}$ \\
\hline $\begin{array}{l}\mathrm{A} \\
\mathrm{T}\end{array}$ & $\begin{array}{l}1 \\
0\end{array}$ & $\begin{array}{l}0 \\
0\end{array}$ & $\begin{array}{l}1 \\
8\end{array}$ & $\begin{array}{l}\text { Fire } \\
\text { protection of } \\
\text { metal } \\
\text { structures }\end{array}$ & & $\begin{array}{c}2,100 \\
.23\end{array}$ & $\mathrm{~m} 2$ & 935.00 & 860.00 & 890.00 & 895.00 \\
\hline & & & & $\begin{array}{l}\text { Total for the } \\
\text { section }\end{array}$ & & & & $\begin{array}{c}225,494 . \\
11\end{array}$ & $\begin{array}{c}242,580 \\
00\end{array}$ & $\begin{array}{c}197,926 . \\
00\end{array}$ & $\begin{array}{c}222,00 \\
0.04\end{array}$ \\
\hline
\end{tabular}

At the second stage, a meeting with each of the contestants takes place and the average price based on the results of the competition is shown and it is offered to the contestant if his price was below the average, then leave his rate, if the price of the contestant is above the average, then reduce it to the average or minimum price (Table 6).

Table 6. Stage II tender results.

\begin{tabular}{|c|c|c|c|c|c|c|c|c|c|c|}
\hline 1 & 2 & 3 & 4 & $\begin{array}{l}\text { Name of work } \\
\text { elements }\end{array}$ & $\begin{array}{c}\text { qty } \\
\text { pe } \\
r \\
\text { uni } \\
t\end{array}$ & qty & $\begin{array}{c}\text { unit } \\
\text { s } \\
\text { rev }\end{array}$ & $\begin{array}{c}\text { Competito } \\
\text { r's rate } \\
\text { No. } 1 \\
\text { (excluding } \\
\text { VAT) }\end{array}$ & $\begin{array}{c}\text { Average } \\
\text { price } \\
\text { per item } \\
\text { (excludi } \\
\text { ng } \\
\text { VAT) }\end{array}$ & $\begin{array}{c}\text { The final } \\
\text { price of } \\
\text { the } \\
\text { participa } \\
\text { nt No. } 1 \\
\text { (excludi } \\
\text { ng VAT) }\end{array}$ \\
\hline $\begin{array}{l}\mathbf{A} \\
\mathbf{T}\end{array}$ & & & & $\begin{array}{l}\text { SURFACE } \\
\text { CONSTRUCTI } \\
\text { ONS (above } \\
0.00) \\
\end{array}$ & & & & & & \\
\hline $\begin{array}{l}\mathrm{A} \\
\mathrm{T}\end{array}$ & $\begin{array}{l}1 \\
0\end{array}$ & & & $\begin{array}{l}\text { Bearing } \\
\text { structures }\end{array}$ & & & & & & \\
\hline $\begin{array}{l}\mathrm{A} \\
\mathrm{T}\end{array}$ & $\begin{array}{l}1 \\
0\end{array}$ & $\begin{array}{l}0 \\
0\end{array}$ & & $\begin{array}{l}\text { Construction part } \\
\text { above } 0.00\end{array}$ & & & & & & \\
\hline $\begin{array}{l}\mathrm{A} \\
\mathrm{T}\end{array}$ & $\begin{array}{l}1 \\
0\end{array}$ & $\begin{array}{l}0 \\
0\end{array}$ & $\begin{array}{l}0 \\
1\end{array}$ & $\begin{array}{l}\text { Installation of } \\
\text { the metal frame } \\
\text { of the building }\end{array}$ & & 203.33 & $\mathbf{t}$ & $24,250.00$ & $\begin{array}{r}24,916.6 \\
7\end{array}$ & $\begin{array}{r}24,250.0 \\
0\end{array}$ \\
\hline $\begin{array}{l}\mathrm{A} \\
\mathrm{T}\end{array}$ & $\begin{array}{l}1 \\
0\end{array}$ & $\begin{array}{l}0 \\
0\end{array}$ & $\begin{array}{l}0 \\
2\end{array}$ & $\begin{array}{l}\text { - metal frame } \\
\text { according to the } \\
\text { project }\end{array}$ & $\begin{array}{r}1.0 \\
0\end{array}$ & 203.33 & $\mathbf{t}$ & $83,050.85$ & $\begin{array}{r}87,572.2 \\
8\end{array}$ & $\begin{array}{r}81,666.0 \\
0\end{array}$ \\
\hline
\end{tabular}




\begin{tabular}{|c|c|c|c|c|c|c|c|c|c|c|}
\hline $\begin{array}{l}\text { A } \\
\text { T }\end{array}$ & $\begin{array}{l}1 \\
0\end{array}$ & $\begin{array}{l}0 \\
0\end{array}$ & $\begin{array}{l}0 \\
3\end{array}$ & Building roofing & & $\begin{array}{r}4,535 . \\
00\end{array}$ & m2 & $3,783.00$ & $3,835.00$ & 287.00 \\
\hline $\begin{array}{l}\mathrm{A} \\
\mathrm{T}\end{array}$ & $\begin{array}{l}1 \\
0\end{array}$ & $\begin{array}{l}0 \\
0\end{array}$ & $\begin{array}{l}0 \\
4\end{array}$ & $\begin{array}{l}\text { Monolithic } \\
\text { interfloor slab } \\
\text { device }\end{array}$ & & 38.40 & m3 & $7,566.00$ & $7,488.00$ & $7,098.00$ \\
\hline $\begin{array}{l}\mathrm{A} \\
\mathrm{T}\end{array}$ & $\begin{array}{l}1 \\
0\end{array}$ & $\begin{array}{l}0 \\
0\end{array}$ & $\begin{array}{l}0 \\
5\end{array}$ & $\begin{array}{l}\text { - Concrete B20, } \\
\text { grade according } \\
\text { to the project }\end{array}$ & $\begin{array}{r}1.0 \\
2\end{array}$ & 39.17 & $\mathrm{~m} 3$ & $5,529.00$ & $5,509.67$ & $5,300.00$ \\
\hline $\begin{array}{l}\mathrm{A} \\
\mathrm{T}\end{array}$ & $\begin{array}{l}1 \\
0\end{array}$ & $\begin{array}{l}0 \\
0\end{array}$ & $\begin{array}{l}0 \\
6\end{array}$ & $\begin{array}{l}\text { - Fittings, } \\
\text { according to the } \\
\text { project }\end{array}$ & $\begin{array}{r}0.0 \\
7\end{array}$ & 2.50 & $\mathrm{t}$ & $42,412.50$ & $\begin{array}{r}43,470.8 \\
3\end{array}$ & $\begin{array}{r}42,412.5 \\
0\end{array}$ \\
\hline $\begin{array}{l}\mathrm{A} \\
\mathrm{T}\end{array}$ & $\begin{array}{l}1 \\
0\end{array}$ & $\begin{array}{l}0 \\
0\end{array}$ & $\begin{array}{l}0 \\
7\end{array}$ & $\begin{array}{l}\text { Installation of } \\
\text { wall panels, } \\
\text { including } \\
\text { additional } \\
\text { elements }\end{array}$ & & $\begin{array}{r}4,272 . \\
40\end{array}$ & m2 & $1,100.00$ & $1,100.00$ & $1,000.00$ \\
\hline $\begin{array}{l}\mathrm{A} \\
\mathrm{T}\end{array}$ & $\begin{array}{l}1 \\
0\end{array}$ & $\begin{array}{l}0 \\
0\end{array}$ & $\begin{array}{l}0 \\
8\end{array}$ & $\begin{array}{l}\text { - wall panels of } \\
\text { the sandwich } \\
\text { type, thickness } \\
80 \mathrm{~mm}\end{array}$ & $\begin{array}{r}1.1 \\
0\end{array}$ & $\begin{array}{r}4,699 . \\
64\end{array}$ & $\mathrm{~m} 2$ & $1,697.50$ & $1,692.50$ & $1,627.50$ \\
\hline $\begin{array}{l}\text { A } \\
\text { T }\end{array}$ & $\begin{array}{l}1 \\
0\end{array}$ & $\begin{array}{l}0 \\
0\end{array}$ & $\begin{array}{l}0 \\
9\end{array}$ & $\begin{array}{l}\text { - additional } \\
\text { elements }\end{array}$ & $\begin{array}{r}0.6 \\
3\end{array}$ & $\begin{array}{r}2,960 . \\
77\end{array}$ & $\mathrm{~m} 2$ & 824.50 & 818.17 & 782.00 \\
\hline $\begin{array}{l}\mathrm{A} \\
\mathrm{T}\end{array}$ & $\begin{array}{l}1 \\
0\end{array}$ & $\begin{array}{l}0 \\
0\end{array}$ & $\begin{array}{l}1 \\
0\end{array}$ & $\begin{array}{l}\text { Installation of } \\
\text { PVC window } \\
\text { blocks }\end{array}$ & & 273.60 & m2 & 508.47 & 522.82 & 461.54 \\
\hline $\begin{array}{l}\mathrm{A} \\
\mathrm{T}\end{array}$ & $\begin{array}{l}1 \\
0\end{array}$ & $\begin{array}{l}0 \\
0\end{array}$ & $\begin{array}{l}1 \\
1\end{array}$ & -block window & $\begin{array}{r}1.0 \\
0\end{array}$ & 273.60 & m2 & $4,237.29$ & $4,712.43$ & $4,237.29$ \\
\hline $\begin{array}{l}\mathrm{A} \\
\mathrm{T}\end{array}$ & $\begin{array}{l}1 \\
0\end{array}$ & $\begin{array}{l}0 \\
0\end{array}$ & $\begin{array}{l}1 \\
2\end{array}$ & Can door blocks & & 10.50 & $\mathrm{~m} 2$ & 500.00 & 500.00 & 450.00 \\
\hline $\begin{array}{l}\mathrm{A} \\
\mathrm{T}\end{array}$ & $\begin{array}{l}1 \\
0\end{array}$ & $\begin{array}{l}0 \\
0\end{array}$ & $\begin{array}{l}1 \\
3\end{array}$ & -block door & $\begin{array}{r}1.0 \\
0\end{array}$ & 10.50 & m2 & $12,000.00$ & $\begin{array}{r}11,166.6 \\
7\end{array}$ & $\begin{array}{r}10,500.0 \\
0\end{array}$ \\
\hline $\begin{array}{l}\mathrm{A} \\
\mathrm{T}\end{array}$ & $\begin{array}{l}1 \\
0\end{array}$ & $\begin{array}{l}0 \\
0\end{array}$ & $\begin{array}{l}1 \\
4\end{array}$ & Gate can & & 108.00 & $\mathrm{~m} 2$ & $1,000.00$ & $1,006.67$ & 970.00 \\
\hline $\begin{array}{l}\mathrm{A} \\
\mathrm{T}\end{array}$ & $\begin{array}{l}1 \\
0\end{array}$ & $\begin{array}{l}0 \\
0\end{array}$ & $\begin{array}{l}1 \\
5\end{array}$ & $\begin{array}{l}\text { - gate according } \\
\text { to the project }\end{array}$ & $\begin{array}{r}1.0 \\
0\end{array}$ & 108.00 & $\mathrm{~m} 2$ & $28,000.00$ & $\begin{array}{r}19,966.6 \\
7\end{array}$ & $\begin{array}{r}28,000.0 \\
0\end{array}$ \\
\hline $\begin{array}{l}\mathrm{A} \\
\mathrm{T}\end{array}$ & $\begin{array}{l}1 \\
0\end{array}$ & $\begin{array}{l}0 \\
0\end{array}$ & $\begin{array}{l}1 \\
6\end{array}$ & $\begin{array}{l}\text { Installation of } \\
\text { internal walls } \\
\text { and partitions }\end{array}$ & & 259.20 & $\mathrm{~m} 2$ & $3,600.00$ & $3,716.67$ & $3,600.00$ \\
\hline $\begin{array}{l}\mathrm{A} \\
\mathrm{T}\end{array}$ & $\begin{array}{l}1 \\
0\end{array}$ & $\begin{array}{l}0 \\
0\end{array}$ & $\begin{array}{l}1 \\
7\end{array}$ & Finishing work & & 192.00 & $\mathrm{~m} 2$ & $4,500.00$ & $3,110.00$ & 430.00 \\
\hline $\begin{array}{l}\mathrm{A} \\
\mathrm{T}\end{array}$ & $\begin{array}{l}1 \\
0\end{array}$ & $\begin{array}{l}0 \\
0\end{array}$ & $\begin{array}{l}1 \\
8\end{array}$ & $\begin{array}{l}\text { Fire protection } \\
\text { of metal } \\
\text { structures }\end{array}$ & & $\begin{array}{r}2,100 . \\
23\end{array}$ & $\mathrm{~m} 2$ & 935.00 & 895.00 & 860.00 \\
\hline & & & & $\begin{array}{l}\text { Total for the } \\
\text { section }\end{array}$ & & & & $225,494.11$ & $\begin{array}{r}222,000 . \\
04 \\
\end{array}$ & $\begin{array}{r}213,931 . \\
83 \\
\end{array}$ \\
\hline
\end{tabular}

The contract for the execution of works must be concluded at the minimum price of the second stage of the competition.

Thus, the formation of tender procurement documentation for placement on trading floors should be carried out using this classification as part of the ordering strategy implemented by the customer.

The success of construction depends on how correctly the choice of the strategy for placing orders was made, how the optimal ratio of time, cost and quality of work, services or supplies was chosen. 
This technique is actively used in foreign countries and can be adapted for Russian conditions, taking into account the territorial and industry specifics.

According to the UK Treasury Department, the optimal ordering strategy will reduce the final cost of the property by $10-15 \%$, and the delivery time - by $50-70 \%$.

\section{Conclusion}

The use of new methods of forming a price proposal for the implementation of construction creates effective conditions for optimizing costs at the stage of their planning.

The detailing of costs by various items in the context of various service providers allows, on the basis of a comparative analysis, to determine the minimum unit costs and identify reserves for their reduction without reducing the regulatory profit of the organization.

This method allows disclosing the actual costs of the contractor and avoiding the "shadow" economy.

The use of resource pricing technologies in construction projects and the systematization of types of construction work at various stages of construction production also allows laying the foundation for assessing the digital maturity of enterprises, which is an important condition for the competitiveness of businesses today (8).

The implementation of such a technology should be carried out on the basis of a change in the structure of the request for proposals and the introduction of a set of documentation templates into the tender activities of the company - the customer or the general contractor, allowing to optimize and unify the procedure for submitting a tender.

The result of this will be the minimization of the costs of contract work for both the customer and the contractor, the customer receiving the required amount of completed construction work of the required quality within the planned time frame.

\section{References}

1. E. Belyaeva, Economic Journal 6-19 (2019)

2. I. Sorokina, International research journal 51-53 (2018)

3. L. Brezgina, The mechanism for managing the cost of investment and construction projects at the stage of contract bidding. Dis. for the degree of Candidate of Economic Sciences (Yekaterinburg, 2017)

4. M. Demidenko, Development of contract bidding for the construction of facilities in the public procurement system. Dis. for the degree of Candidate of Economic Sciences $(\mathrm{SPb}, 2017)$

5. A. Lobanova, Scientific format (2019)

6. I. Artamonova, A. Chernitsov, D.Osipov, A.Tuskov, A. Konkin, J. Ponte 73-2 (2017)

7. https://www.uniformat.com/

8. P. Bilenko, S. Lysenko, I. Zavaleev, L. Lysenko, Scientific Technologies 7 (2017) 